\title{
Operating Experiences with the Service Robot MOPS
}

\author{
N. Tschichold-Gürman, S. J. Vestli, G. Schweitzer \\ Institute of Robotics, ETH Swiss Federal Institute of Technology, CH-8092 Zurich \\ \{tschichold, vestli, schweitzer\}@ifr.mavt.ethz.ch
}

\begin{abstract}
The Mobile Post Distribution system MOPS has been developed at the ETH Zurich during the last few years. Numerous technical problems had to be solved during this period. Currently, we are operating and testing it in our office building. A service robot like MOPS is a highly complex system, as it includes, in addition to the robot itself, the task, the operator, and the environment. Operating experiences with such service robots are not easily available yet. They would be of value, as, in the end, customer acceptance will decide upon the actual use of service robots.

In this paper, technical aspects of the realization of MOPS are discussed briefly. Furthermore, operating experiences, lessons learned and consequences are commented.
\end{abstract}

\section{Introduction}

Service robots are being suggested for a growing number of applications [13]. Their ability of being mobile spurs the imagination of many potential users. However, operating experiences with service robots are not easily available yet [5], [7]. They would be of value, as, in the end, customer acceptance will decide upon the actual use of service robots, and future research would have to be stimulated into reducing deficiencies coming up during operation.

A service robot is a complex system, as it includes, in addition to the robot itself, the task, the operator, and the environment. Its performance is measured with respect to technical specifications but also with respect to expectations of skilled operators and even of unspecified users. In our case, as a realistic test platform for investigating a complex mobile robot application, we have been developing the Mobile Post Distribution System MOPS (Fig. 1). The robot has the task to transport boxes of mail between the central mail room and the secretaries' offices which are distributed on five floors. Currently we are testing and operating it in our office building. To realize this mobile robot application, we have addressed several fundamental mobile robot research problems. In this paper the technical aspects, like navigation and control principles as well as the natural landmark recognition based position update strategy are briefly described.

Further, the paper addresses the interaction of the robot with its environment and the users. The operating experi- ence, main causes for disturbances or malfunctions, as well as consequences for the actual layout and operation of the robot are discussed. An outlook on future research topics, which we think should be tackled, are also given.

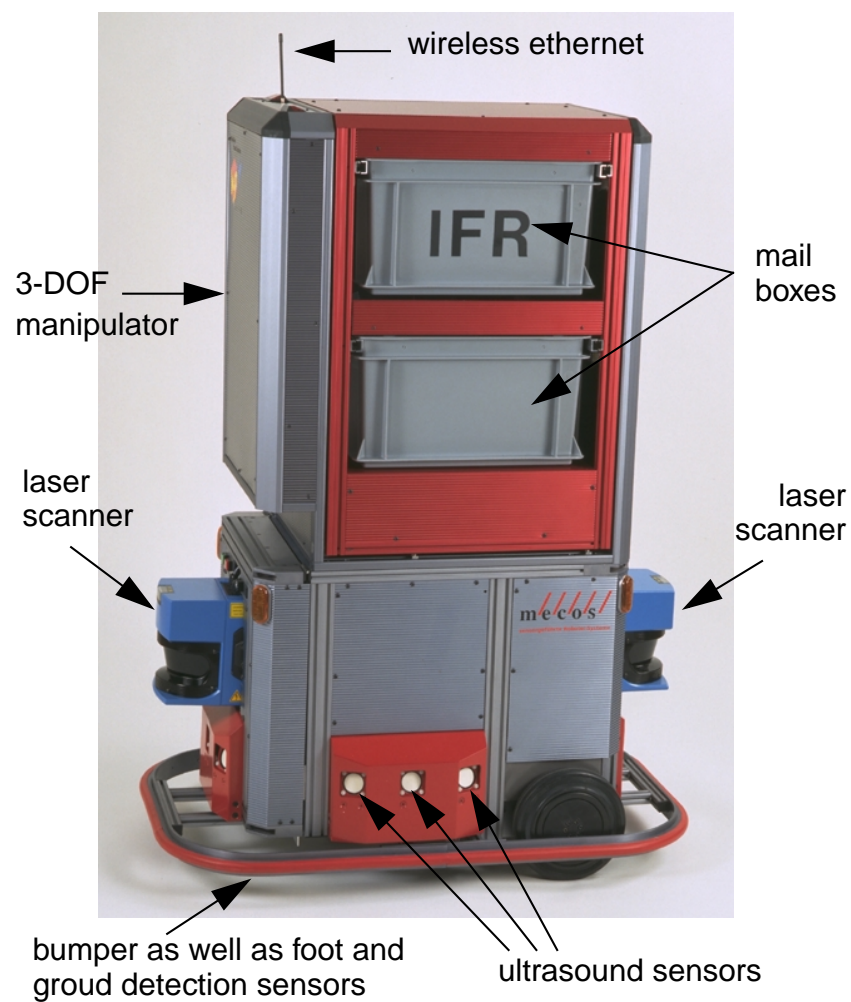

Fig. 1. The MObile Post System MOPS. The upper part of the system constitutes a 3-DOF manipulator / storage mechanism for two mail boxes. The lower part is a mobile robot platform with two drive and one castor wheels.

\section{Task Description}

In our institute building at the Swiss Federal Institute of Technology in Zurich (ETH) there are several departments each requiring separate internal mail distribution. Currently, mail distribution in this $150 \mathrm{~m} * 15 \mathrm{~m}$ building is done manually.

The departments are spread on 5 floors within the building and each department has up to two secretariats located 
more or less centrally to that particular department. Twice a day the mail arrives in a mail room centrally in the building and the mail for each institute is put into mail boxes, one for each department. These and the boxes with outgoing mail are then placed in pigeon-holes on loosely guided rails. Currently, somebody from each institute then picks up the institute's mail from the mail room and also deposits the institute's outgoing mail in the corresponding pigeon-hole. The robot's task is to relieve the members of the institute of the daily "pilgrimage" to the central mail room.

Thus, the robot picks up the boxes at any of these mail rooms and deposit them at one of the other mail rooms. Since this is a public building with unlimited access, protection against theft of the mail must be provided. This has been realized with motorized blinds for the pigeon-holes, blinds which can be opened by the robot, the secretaries and the postal service.

\section{Robot System}

For the task described above a mobile robot platform with non-holonomic kinematics, two diametrically opposed drive wheels and a free-wheeling castor, is being used. The two drive wheels are located at one end (the front end) of the vehicle, and the support castor at the other end.

The robot system contains a multi-processing controller for servo motors (DC, EC or AC) based around the VME bus. Currently the robot is equipped with one $200 \mathrm{MHz}$ PowerPC 604 processing board. The drive wheels constitute two servo axes, and the remaining 4 axes are available for the box manipulating mechanism on top of the platform. The programming is done in the object-oriented real-time development environment XOberon for general, high-performance mechatronic systems [4], [17].

The basic robot platform is equipped with two scanning laser range-finders (LIDARs), the LMS200 from the company SICK, and a bumper system with six segments. For security purposes, twelve ultrasonic range finders are additionally placed around the robot. Further laser distance sensors are used for improving the precision of the robot system in critical docking maneuvers, for example when the robot aligns itself for the tasks of mailbox manipulation or automatic battery exchange. A wireless ethernet system enables the communication of the robot with the intranet of the institute as well as with Internet. Various indicator lights and a speech output module inform the environment about the robot's immediate action. An infra-red communication system is used to communicate with the building infrastructure (elevator, pigeon hole blinds, etc.). A touch-screen-PC is mounted on the robot so as to provide an ergonomic user interface.

\section{Navigation and Control}

After a task is defined by the user, e.g. drive to the central mail room, the global planner generates a list of the intermediate positions that have to be passed to reach the final goal. This plan is calculated using the map of the robot's operation environment (see 4.1 for more details). The navigation behavior of the robot on each of these path segments is then selected by a situation based behavior selector (SBBS), which is discussed in 4.2. The different behaviors are implemented independently (section 4.3). Most of these behaviors are based on a position controller, which is discussed in section 4.4 .

\subsection{Global Planner}

When the operating environment of the robot is known and represented in a graph structure, global planning can be realized using a graph search algorithm. Therefore, the operation environment of the MOPS is stored in a generic, directed graph. Each graph node contains the world coordinates of that point and the walls that can be seen by the robot at that position.

Further information can also be stored in the graph edges which are associated to path segments. This allows us to define the behavior as well as the reactions of the robot while traversing a particular segment. Thus the possible algorithms to be applied on a single path segment are defined by the user.

Operating on this graph, the global planner generates a list of intermediate path segments to be visited to reach a goal position. The local planner takes the next path segment from the list and tries to reach it using the information associated to this list element.

For the planning of the robot's travel route, several graph search algorithms can be used. The graph representing an office building as described above is sparse (usually two, maximal four edges per node) and small (a few thousands nodes), therefore the breadth search algorithm [14] can be used, which always delivers optimal routes and it is efficient enough to be computed in real-time. It is also possible to use a weighted graph, to mark edges which are difficult to travel (e.g. routes that are usually crowded). In this case, a Priority-First Search algorithm can be used [14]. The use of a weighted graph is recommended in buildings with more than one corridor, enabling different routes to reach a location. In the case of MOPS, a weighted graph has been implemented with the Priority-First Search algorithm to have a solution which can also be used in complex buildings.

\subsection{A Situation Based Behaviour Selector (SBBS)}

As mentioned in the previous section, the global planner generates a list of the path segments to be traversed by the robot to reach a predefined goal. The situation based behav- 
ior selector (SBBS) is responsible for scheduling the appropriate navigation algorithms as indicated by the information contained in the path segment data packet and the current sensor data (Fig. 2).

The standard case is that the subgoals defined by the global planner can be reached on simple trajectories, often straight lines. Thus the robot must be able to identify its position in the world coordinate system and update it continuously (see section 5). It is then an easy task to travel on the desired trajectory using classical control techniques, e.g. a position controller (see section 4.4). However, in a real world environment the path defined by the global planner may be impassable at certain times, e.g. the robot has to drive around an obstacle. Thus the robot has to recognize the situation and react properly.

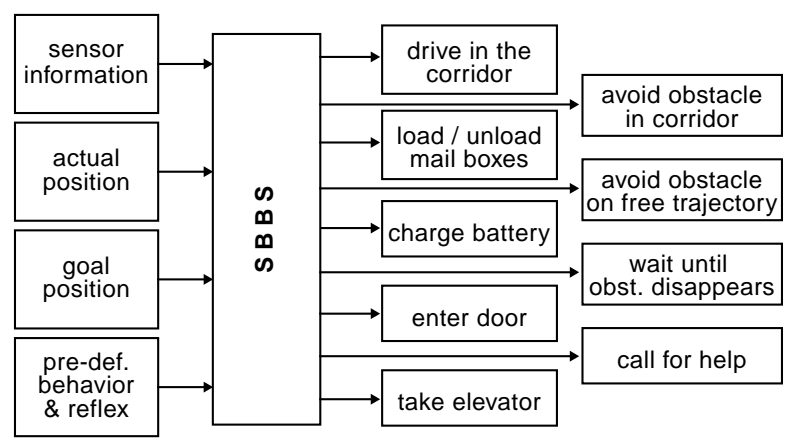

Fig. 2. The situation based behaviour selector, associated behaviours on the right and input information on

The SBBS utilizes two dimensional range data stemming from two laser range sensors (section 3) in order to recognize situations for the mobile robot. A situation is defined as a recognized scene within an environment, if measurements of the sensors at a pre-defined position of the robot correspond to a set of measurements memorized before. Recognized situations are then used as a basis to provide a certain behavior (a particular algorithm competent in handling the situation) for the mobile robot. For the current application, the SBBS consists of simple rules that operate on environmental information introduced into the graph manually, however it is also possible to use a neural network (e.g. RuleNet) to make the system more independent from the environment [15].

The implementation of the different behaviors can be realized using different algorithms, including neural, fuzzy and classical control techniques. The main advantage of choosing such a modular control architecture is the possibility of an easy extension of the system. As the implementation of the behavior modules are independent of each other, it is possible to change or improve a particular one without affecting the others. It is also easy to add new behavior modules to the system; only the behavior selector module has to be extended and the user can introduce this new behavior in the path segments of the global planner. All the existing behavior modules are not influenced by this extension. On the other hand, if one single module would have to handle several behaviors, changing one behavior could have an uncontrollable influence on other behaviors.

\subsection{Implementation Principle of the Behaviours}

MOPS has mainly two classes of behaviors. The first class allows the robot to travel around in most places in the building, such as in the corridors. The implementation of these behaviors is based mainly on the position controller. If an obstacle is detected by the sensors, a corresponding reaction behavior is selected, e.g. drive around the obstacle in large hallways. In case of narrow corridors, the robot has to stop its movement, give some warning using the speech output; after a while, if the obstacle doesn't change its position, MOPS sends an email to the maintenance office informing about the situation and continues the mail distribution task with the next order, if possible.

Behaviors belonging to the second class are specialized to master specific tasks using additional sensors, e.g. docking maneuver to manipulate the mail boxes or to charge the batteries, taking the elevator, opening the doors, etc. The docking maneuver consists of four sub-behaviors, using (additionally to the LIDARs) the infrared triangulation sensors for exact positioning and the infrared communication system to operate the blinds in front of the racks containing the mail boxes.

In the case of the elevator behavior, the LIDAR sensors are used to detect the state of the elevator doors; the manipulation of the elevator (selecting the floor, getting the floor information, etc.) is realized with the infrared communication system. The design of the interaction strategy of MOPS with people is especially essential during the use of the elevator. The speech output telling the state and the planned actions of MOPS is very important to inform the persons using the elevator. In case of people standing in the door of the elevator and not realizing that the "box" in front of the door is a robot trying to get into the elevator, the robot must be able to recognize the situation and tell the people to step aside. If there is no space in the elevator, or the obstacle in the elevator is a large object, the robot must decide to wait for the next elevator. To make this decision, a vision system is currently in development, which can detect the presence of humans and which thus can distinguish between people and obstacles in the elevator. Additionally, MOPS informs the environment about its state and intentions using various indicators. A further important feature of MOPS is to send an email to the maintenance office in an exceptional situation, for example if the elevator doesn't come, after waiting a specified time. It can happen that a person forgets to close 
the elevator door (which are operated manually in some floors). In this case, the elevator and consequently the robot are blocked. Thus, the robot would wait in front of the elevator, until its batteries get empty (unless it is currently on the floor where the charging station is located). So it is essential that a communication to responsible people is guaranteed. A further example of a behavior, namely the box handling, is depicted in Fig. 3.

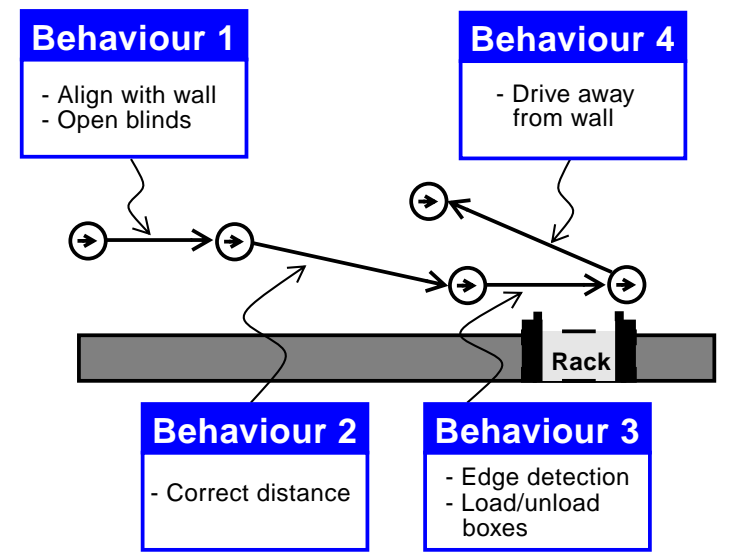

ig. 3. Schematic view of the docking behaviour.

As shown in the above examples, the autonomy of such mobile service robots is always limited. The interactions between robot and people have to be designed carefully. Some additional details of this topic are discussed in section 6.

\subsection{Position Controller}

In the previous sections we have described how the MOPS performs global and local navigation. One step of the navigation involves generating a sequence of attitudes ( $\mathrm{x}, \mathrm{y}$ and $\theta$ ) that should be visited, one after the other, by the robot (see global planner in 4.1). To enable the non-holonomic MOPS to execute such trajectories composed of a number of attitudes a position controller has been developed.

The position controller employed for the MOPS project is an improvement of the controller described in [1]. This position controller transforms the Cartesian error vector, $\left[e_{x}\right.$, $\left.e_{y}, e_{\phi}\right]$, in the robot coordinate system into its equivalent polar representation, $[\alpha, \rho, \phi]$, by means of equations (1) to (3). It is worth noting that equation (1) is undefined in the goal point, thus the controller must switch mode as the error decreases below a certain threshold.

$$
\begin{gathered}
\alpha=\operatorname{atan} 2\left(e_{y}, e_{x}\right) \\
\rho=\sqrt{\left(e_{x}\right)^{2}+\left(e_{y}\right)^{2}} \\
\phi=e_{\phi}
\end{gathered}
$$

With the above transformation the feedback controller given by equations (4) and (5), where $v$ is the command translational velocity of the robot and $\omega$ is the command rotational velocity, ensures highly accurate positioning.

$$
\begin{gathered}
v=k_{p} f(\rho) g(t) \\
\omega=\left(k_{\alpha} \alpha+k_{\phi} \phi\right) g(t)
\end{gathered}
$$

The current implementation of the position controller is able to reach a given goal point within an accuracy of less than $1 \mathrm{~mm}$ and $0.1^{\circ}$, see also the trace in Fig. 4. Such high accuracy is only possible thanks to the stiff velocity controllers (all controllers are realized as software processes under XOberon) which achieve a step input response time delay of $0.1 \mathrm{~s}$. Further details on this position controller can be found in [9]. This controller ensures that the MOPS follows the planned trajectories and avoidance maneuvers, and its accuracy is high enough to enable reliable docking at the pigeon holes.

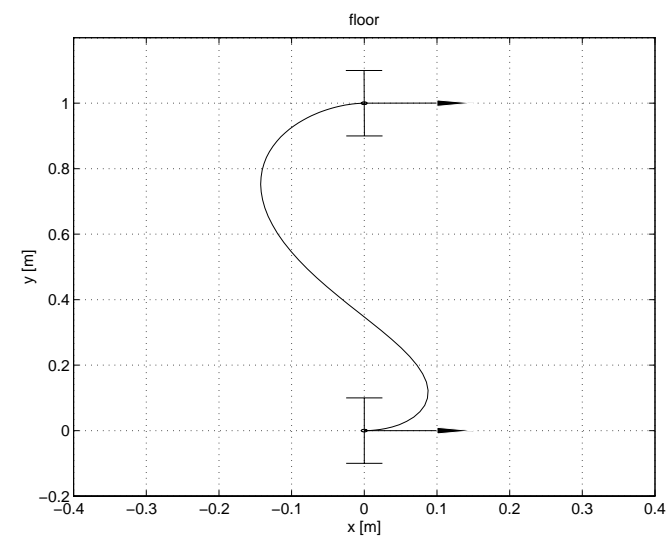

Fig. 4. Trace of the robot $x$ and $y$ world co-ordinates for a one metre translation in (robot) y direction.

\section{Position Update}

Unless specific measures are taken a mobile robot does not automatically have any knowledge of its own position. Due to the incremental nature of dead reckoning it is inevitable that even small systematic errors such as those resulting from inaccurate knowledge of the wheel radii will over time add together and take on unacceptable proportions.

Thus a position update method has to be integrated that provides real time performance, high accuracy, and robustness. In order to achieve high estimation accuracy, consequent propagation of the first and second statistical moments from single range reading to all system stages involved in the position estimation is essential. Extraction of geometrical primitives, such as walls corners, cylinders and artificial retro-reflectors, from noisy $1 \mathrm{D}$ range and intensity data thus has to furnish the first two moments of the model parameter 
estimates. The mathematics behind the position update can be found in [2], where the problem is split into two steps, a feature extraction and a subsequent calculation of the robots position.

Feature extraction can again be subdivided into two steps: $a$ ) determining which of the range measurements in the range image are generated by geometric primitives present in the environment (segmentation / classification), and $b$ ) determining how do theses points contribute to the estimation of the model parameters and their covariance matrix (model fit).

The answer to question b) is, in our case, the estimation of the model parameters in the general least squares sense using polar co-ordinates minimizing the perpendicular distance from the measurement points to the model [1].

Question $a$ ) is a segmentation problem. In this work we employ a probabilistic feature-independent model fidelity criterion in the model space and a subsequent clustering step.

All the models are fitted into $n_{f}$ neighboring points and the covariance matrixes are computed (please note that it is not yet known which of the models considered is the appropriate one for a particular set of neighboring points). This is done for all points of the scan obtaining the same number of spatially diffused Gaussian distributions in the model spaces. When adjacent groups of range readings lie on the same geometrical structure, their associated points constitute a cluster of these distributions in the model space corresponding to that structure (landmark). Feature extraction is now the task of finding these clusters. In a general case a clustering problem of this size where no a priori knowledge is available would lead to impracticably high computation times under real time conditions. Here the fact can be exploited that points on the same landmark are almost always consecutive points.

Due to this underlying regularity in the data acquisition process, a compactness measure, $c_{i}$, in the model space is defined (see [2] for further details on the calculation of $c_{i}$ ). Low $c_{i}$ indicates that the points involved have high model fidelity. If $c_{i}$ is plotted against the measurement index for all $i$, regions of low value can be expected at the corresponding index places of the sought clusters. A threshold $c_{m}$, found by deciding on the appropriate distribution of $c$, is applied cutting off the regions of low distance. A contributing segment is now defined to be the set of measurement points whose representations in the model space satisfy $c_{i}<c_{m}$.

After the completion of this step there will be, in general, more than one segment corresponding to one single landmark. We have an oversegmented range image. Associating these segments with each other is done in a clustering step. The clustering must ensure that two landmarks which differ only slightly in one or more of their model parameters, i.e. in their position, should be identified as being dis- tinct within the limits which are given by sensor noise. We use an agglomerative hierarchical clustering algorithm [10] which permits an efficient implementation.

Otherwise valuable information would be lost, for example in a matching procedure of a Kalman filter-based localization task, where only the observed segment which is closest to the prediction will be considered, whereas the others belonging to the same landmark would be ignored. A typical processed sensor data scan after segmentation and clustering is shown in Fig. 5.

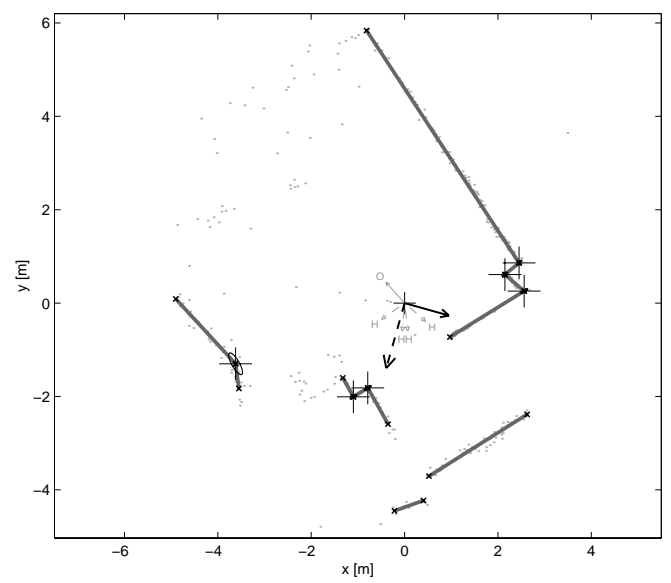

Fig. 5. Extracted lines after the agglomerative hierarchical clustering. Corners which are found according to the scheme in [1] are depicted by crosses and their 99\%

After the clustering step the least squares model-parameter estimation is re-applied, this time to those points that have been identified as lying on a particular geometric structure (landmark). This step yields optimal estimation of the first and second statistical moments of the geometric structures as seen in the robot (sensor) co-ordinate system.

The knowledge of the statistical moments of geometric structures found in the sensor data is used together with a world model (WM) and a Kalman filter based localization scheme to estimate the robots attitude ( $\mathrm{x}, \mathrm{y}$ and $\theta$ ) in the global co-ordinate system. The WM is stored in the navigation graph, see also section 4.1. The robots attitude is used throughout the robot control system, see for example section 4.4 , thus a high quality attitude estimate is essential for reliable autonomous robot operation.

Testing the complete localization method on data as found in Fig. 5 the standard deviation in $\mathrm{x}$ and $\mathrm{y}$ was observed to be $6 \mathrm{~mm}$, whereas standard deviation in $\theta$ was $0.05^{\circ}$. The average localization cycle time during a journey through lab and corridors was $445 \mathrm{~ms}$ (on the previous MC680x0 based controller). Recent experiments on the current MOPS hardware leads us to expect that this over all processing time will be lower by a factor of 50 (i.e. around $10 \mathrm{~ms})$. 


\section{Interaction with Users}

The interaction with humans takes place on various levels. A direct interaction with MOPS is possible via the touchscreen-PC placed on the robot (Fig. 6).

A further interface to MOPS is available on the internet. Several services and information are offered to the public, e.g. the current position of MOPS, information about the building and people working in the building. There are also protected services, such as the possibility for the secretaries to remove themselves from the mail distribution list of MOPS (in case of vacation, etc.). The basic design of these user interfaces is the result of a project with the Institute for Work and Organizational Psychology at the ETH Zurich [8].

Further interaction of MOPS with the environment takes place using the indicator lights and the speech output module. The indicators are placed on four corners of MOPS and signal the current travel direction of the robot. The speech output module is the multi-lingual synthesizer Eurovocs. In specific situations, the robot can use this device to announce information about its actual state or requests to the environment. Additionally, the robot makes position specific comments (e.g. in front of the offices) or tells random wisdom during its normal travel. However, the operating experience with MOPS has shown that this feature annoys the people working in the offices after a certain period (approx. one week) because of the repetitions. A next step will be to load the headline news from the internet incrementally and to tell them during the travel of MOPS.

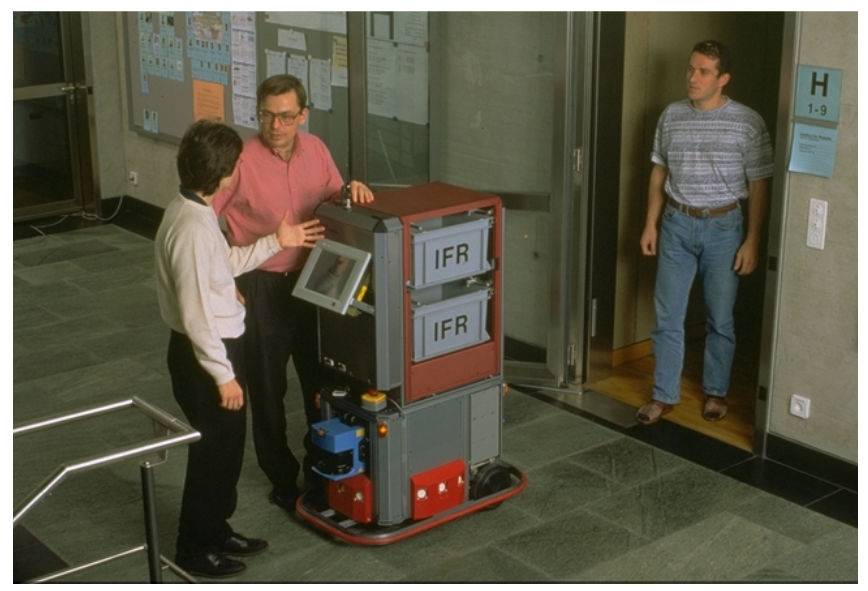

Fig. 6. MOPS driving through populated area.

An important quality of the robot is its acceptance by users. Together with the Institute of Work Psychology of the ETH we made interviews with secretaries, janitors, and students as to the general acceptance of such a new cooperating machine in the building. The answers showed a broad spectrum of attitudes, ranging from enthusiasm to unrealistic high expectations on what such a machine could really do, or to complete rejection [11]. It definitely shows that information and education is a necessary prerequisite, and users should be integrated into making the machine operational. An important aspect as to acceptance is, of course, the widespread feeling that the distribution of mail is still considered as a meaningful human activity which should not be taken over by a soulless machine. This attitude would certainly be different if the work would be a dangerous or unpleasant one.

\section{Collecting Operating Experiences}

Methods of obtaining operational experience vary largely. A log-book of all exceptional events is kept continually. We are discussing causes and consequences in a group, setting up an FMECA approach (Failure Modes, Effects and Criticality Analysis) for improving the system behavior. The service robot MOPS is being operated in an office building, where five institutes of the ETH are located. The introduction of MOPS is realized in several testing phases, followed by appropriate software and hardware revisions, as a consequence of the test results. The length and difficulty grade of MOPS mission is increased for each test period. By now, the following phases have been completed:

- Phase 1: navigation in a single room

- no interaction with building infrastructure, such as doors, elevator, blinds in the front of mail stations

- only a few obstacles (persons) in the room.

- robot takes a mail box continuously from the same rack and puts it back, thus there is no change of box position by human.

- Phase 2: handling of empty mail boxes in a simple tour from the lab to an adjacent mail-stop

- navigation in the corridors of two floors

- full interaction with building infrastructure: doors, elevator and blinds in the front of mail stations

- interaction with people (mostly institute staff)

- Phase 3: mail delivery for one secretary: tour between mail center on the ground floor and one secretary mailstop as well as battery charging station

- interaction with all persons working in the building as well as visitors, students, people challenging the robot, e.g. covering the sensors

- robot takes mail box from the mail center, delivers it to the secretary's mail-stop, takes an empty mail box and delivers it back to the mail center

- interference of people handling the mail boxes at the stops as well

- automated battery management (battery exchange before starting a mail tour)

Currently, the third test period of 21 days has been completed; the robot has been accompanied by a service person (not one of the developers of the system) who assists MOPS 
occasionally and notes the reactions as well as the actions of the people. It is very important to follow MOPS in a far distance to let people "play" with the robot. This experience is needed for the refinement of the exception handling.

The overall success rate of MOPS during the first test phase is $100 \%$ in a total of six hours of operation. However, the results of the third test phase are not satisfactory yet, although the reliability of the robot behavior is very high (the number of collisions is only two). The reasons for non perfect behavior can be grouped in the following six categories:

- Navigational errors, e.g. "ghost" obstacles while maneuvering in narrow passage-ways

- Positioning errors, e.g. due to miss-matching of small wall areas in the presence of many obstacles (in situations with too many people around, robot gets lost)

- Problems while box handling, e.g. no gripping possible of mail box due to its bad positioning; failure of mail box pulling because of incorrectly loaded, overfilled boxes

- Problems with energy management, e.g. empty backup battery which is needed to bridge the time when the main batteries are changed

- Problems while interacting with environment, e.g. malfunctioning automatic doors and blinds

- Network problems, e.g. robot cannot communicate with the battery charging station to give the command to start battery exchange due to overloaded network (Ethernet)

A survey about the problems having occurred during the operation of MOPS during the tours in test phase 3 is shown in Fig. 7. A tour consists of changing the batteries, taking a mail box from the mail center, delivering it to the secretary's mail-stop and bringing an empty box back to the mail center. Note that several errors are possible on a single tour, because service staff is accompanying the robot on its tour and intervening in case of problems, thus the tour can continue after the occurrence of an error situation.

It is interesting to see that the largest number of failures comes from interaction of the robot manipulator with the mail boxes and their holding compartments. It points to a basic problem: these elements are being used and manipulated by humans as well, as boxes are loaded manually and put into their holding compartments, and obviously our robot manipulator is not versatile enough to cope successfully with all of the imperfections caused by human handling. Thus, it is the interfacing of human actions with robot action that causes the problems. We will have to make the robot manipulator more intelligent, or we will have to put constraints on the human handling of the boxes. Customers, in general, tend to prefer an intelligent robot as students from our Department of Production Engineering found out, who had been doing marketing research among potentially large customers in Switzerland and Germany, exploring

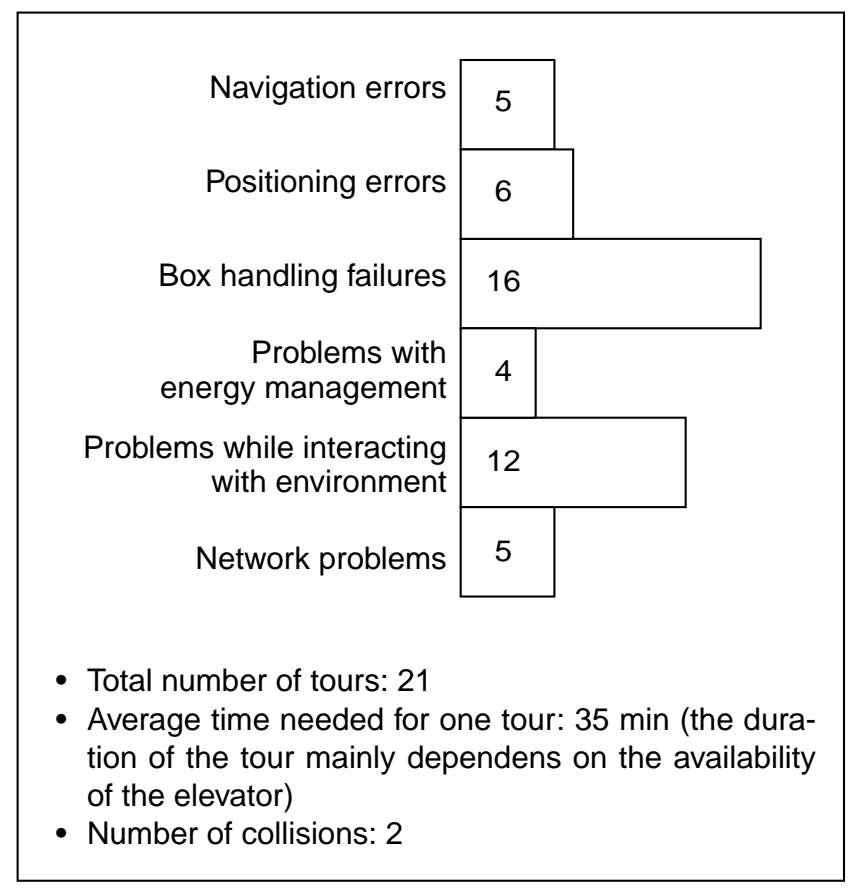

Fig. 7. Survey on problems having occurred during the most advanced test phase 3 of MOPS operation.

their expectations concerning robot behaviors and additional applications [12].

\section{Conclusions and Outlook}

In this paper, the mail distributing service robot MOPS has been presented. Specifically, the robot system, the navigation and control architecture, the position controller, the localization method, and the human-world interface that are in use on the MOPS for its task of distributing the mail at the Swiss Federal Institute of Technology, Zurich, have been described. The robot is picking up boxes at the ground floor of a five floor building, delivering them to the secretaries' offices, subsequently bringing back the outgoing mail to the ground floor station.

The robot is autonomous in the sense that it is freely navigating based on on-board sensor signals. Its navigation is based on the recognition of natural landmarks which are compared to data of the building lay-out stored on the robot's processor. We have shown that the system has a highly accurate localization and position control system, which in turn enables the robot to "dock onto" the pigeon holes and to load/unload boxes of mail. Furthermore the position update process is tolerant towards unmodelled obstacles and the position update is also fast enough for utilization in the real world. The hybrid navigation scheme combining graph-searching with a situation based behavior selector and appropriate behaviors has proved itself as 
robust and efficient. The generated paths are close to being optimal, and the tolerance towards obstacles is high.

The robot is actually in an advanced test phase. The experience with user interaction lead us to support the following approaches:

- The robot should indicate its state in such a way that the user or passer-by can guess what it is going to do next. Voice output was very well accepted.

- People have to become familiar with the co-existence of technical co-workers such as mobile robots. This may require special educational efforts and it certainly will take time. The integration of all future users, in particular the secretaries and the janitors and not only the technical staff, into the introduction of the MOPS operation led to very positive reactions.

As an outlook we will summarize some topics we think would be necessary or useful to further promote the use of such mobile robots:

- Currently the system relies upon an a-priori map. Investigations are being undertaken with the goal of eliminating this costly, and unnecessary step.

- Localization and map building should also be able to use vision for reading door labels with numbers and names. Some promising results have been obtained already [16].

- The robot should have the ability of active self-diagnosis. In case of degradation or failure it should hypothesize on possible error sources and it should actively pursue a strategy to verify its suggestions, i.e. smart machine technology has to be developed. In some cases, this might even allow the machine to switch to corrective measures automatically.

- Currently, obstacle avoidance is done in the conventional way, using the robot's laser range finder and its behavioral navigation. It could be refined, by detecting the presence of humans and making a distinction between them and usual obstacles, in order to make the robot behavior still safer, and maybe, more "polite". Promising results have been obtained already.

Basically, the mobility of the mobile robot is not a purpose in its own. The robot will be used for a task or a process, for example handling or cleaning. In future, the integration of the requirements posed by such tasks into the design of a service robot will need more attention.

\section{Acknowledgement}

We express our gratitude to Ruedi Borer for the hardware integration of several sensors and to Beat Peyer for the development of the infrared sensor system for communication with the environment.

\section{References}

[1] K. O. Arras, R. Y. Siegwart, "Feature Extraction and Scene Interpretation for Map-Based Navigation and Map Building", in Proc. of SPIE, Mobile Robotics XII, vol. 3210, pp. 42-53, 1997.

[2] K. O. Arras, S. J. Vestli, "Hybrid, high-precision localisation for the mail distributing mobile robot system MOPS", in Proc. IEEE Int. Conf. on Robotics and Automation, vol. 4, pp. 3129-34, 1998.

[3] A. Astolfi, "Exponential stabilization of a mobile robot" $3^{\text {rd }}$ European Control Conf., Rome, Italy, 1995.

[4] R. Brega. "A Real-Time Operating System Designed for Predictability and Run-Time Safety" in Proc. of the Fourth Int.. Conf. on Motion and Vibration Control (MOVIC'98), vol. 1, pp. 379-384, 1998.

[5] W. Burgard, A. Cremers, D. Fox, D. Haenel, G. Lakemeyer, D. Schulz, W. Steiner, S. Thrun. "The Interactive Museum Tour-Guide Robot" in Proc. Fifteenth National Conf. on Artificial Intelligence (AAAI'98), Madison, Wisconsin, 1998.

[6] P. Cattin, Human Presence Detector, Internal Report, Inst. of Robotics, ETH Zurich, March 1999.

[7] H. Endres, W. Feiten, G. Lawitzky. Field test of a navigation system: autonomous cleaning in supermarkets. In Proc. IEEE Int. Conf. on Robotics and Automation. vol.2. Leuven, Belgium, May 1998.

[8] P. Erni, D. Ritler, M. Rueegg, C. Rohner, R. Reutemann. "Gestaltung der Benutzeroberflaeche für einen Postroboter". Student Project at the Inst. of Robotics, ETH Zurich, 1995.

[9] O. Kaiser, R. Pfiffner, S. Vestli, A. Astolfi, "Positionsregelung für nichtholonome mobile Roboter" 11. Fachgespräche über autonome mobile Systeme, Karlsruhe, Germany, Nov. 1995 (in German).

[10] L. Kaufman, P. J. Rousseeuw, "Finding Groups in Data: An Introduction to Cluster Analysis", Wiley Series in Probability and Math. Statistics, 1990.

[11] J. Kurth. "Benutzerschnittstelle für MOPS". Diploma Thesis at the Inst. of Robotics, ETH Zurich, 1997.

[12] P. Kuebler. "Marktstudie Deutschland". Project Work at the Dept of Production Eng., ETH Zurich, 1997.

[13] R.D. Schraft, G. Schmierer. Serviceroboter. Springer Verlag, Berlin, 1998.

[14] R. Sedgewick, Algorithms, Addison-Wesley, 1988.

[15] N. Tschichold, "RuleNet - A Knowledge-Based Neural Network Model with Application Examples in Mobile Robotics", Ph. D. Thesis 11356, ETH Zurich, 1995.

[16] N. Tschichold, P. Cattin, "Recognition of Door-Number Plates for Mobile Robot Localisation", in Proc. of 3rd Int. Workshop Neuronale Netze in der Anwendung '98, Magdeburg, Feb. 1998.

[17] www.ifr.mavt.ethz.ch/research/xoberon/index.html 